NISSUNA UMANA INVESTIGAZIONE SI PUO DIMANDARE VERA SCIENZIA

S'ESSA NON PASSA PER LE MATEMATICHE DIMOSTRAZIONI

LEONARDO DA VINCI

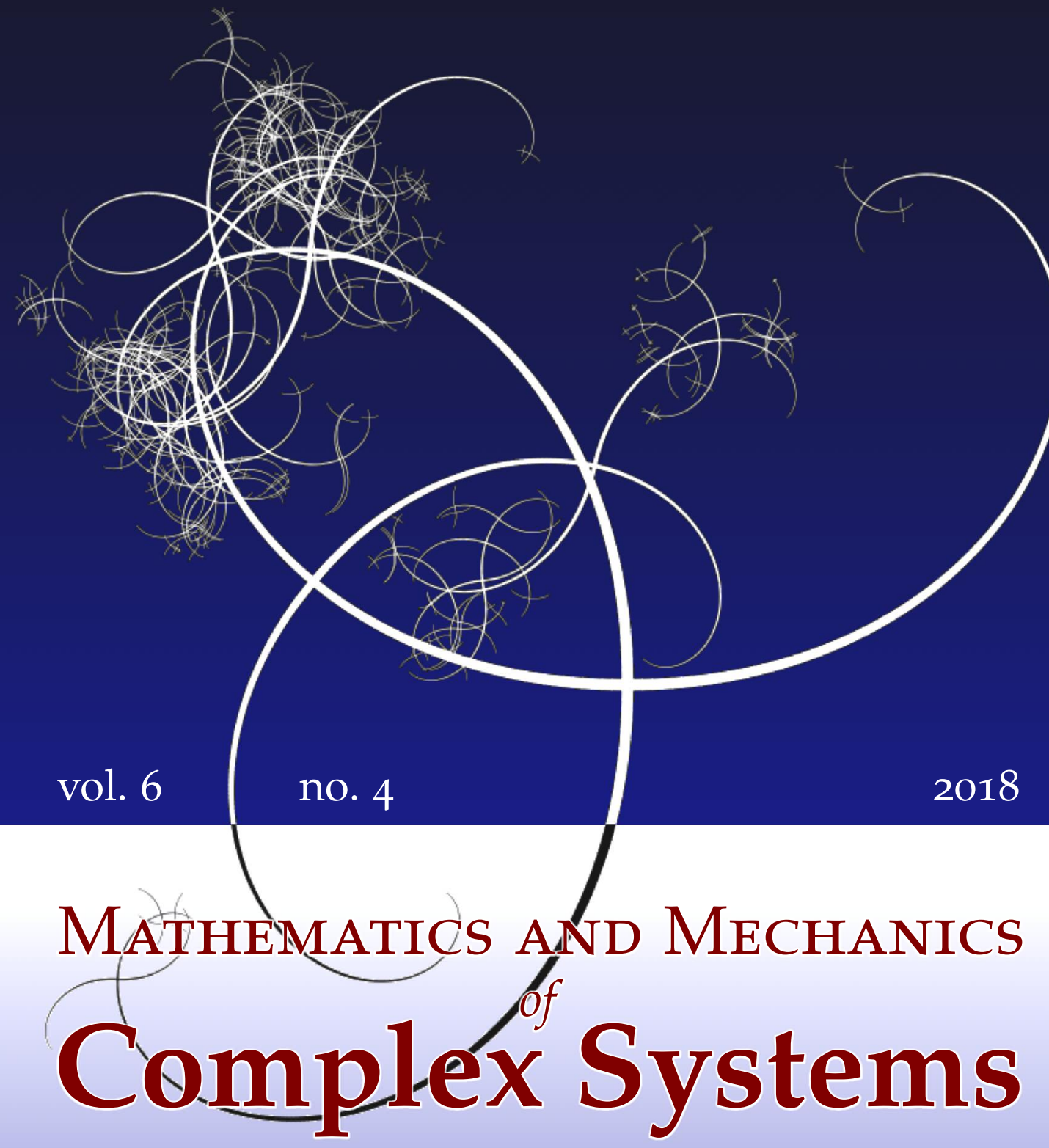

JOE D. GODDARD

ON LINEAR NON-LOCAL THERMO-VISCOELASTIC

WAVES IN FLUIDS 


\title{
ON LINEAR NON-LOCAL THERMO-VISCOELASTIC WAVES IN FLUIDS
}

\author{
JOE D. GODDARD
}

The following is an elaboration on the linear non-local model of viscoelastic fluids proposed in a previous work (Int. J. Eng. Sci. 48 (2010), 1279-1288). As a recapitulation of that work, the basic theory is presented in terms of the temporal frequency and spatial wave number in the Laplace-Fourier domain. Taylor-series expansions in these variables provides a weakly non-local theory in spatio-temporal gradients that is more comprehensive than the "bi-velocity" model of Brenner. The linearized Chapman-Enskog kinetic theory is shown to provide a confirmation of the more general theory, from which one can reconstruct a fully non-local integral model.

Following the work of Davis and Brenner (J. Acoust. Soc. Am. 132 (2012), 2963-2969), the general theory is employed to derive dispersion relations for acoustic, thermal and shear-wave propagation in compressible viscoelastic fluids. At Burnett order the Chapman-Enskog theory gives a cubic polynomial in wave number squared which reduces in the dissipative quasi-static limit to a quadratic like that given by the classical Navier-Stokes-Fourier model and the bi-velocity modification of that model.

With minor modification, the present analysis applies to viscoelastic shear and dilatational wave propagation in solids with higher-gradient and Cosserat effects, where it may, for example, find application to the field of rotational seismology.

\section{Introduction}

Following [Goddard 2010], hereinafter referred to as [G10], we consider a linear, fully non-local model for the thermo-mechanics of fluids. As was the case with [G10], the present paper is motivated in part by the ideas of the late H. Brenner, who wrote extensively at the end of his career [2009; 2012] on the possible breakdown of the classical Navier-Stokes-Fourier model of momentum and heat flux arising

\section{Communicated by Francesco dell'Isola.}

MSC2010: 35Q72, 44A30, 74JXX.

PACS2010: primary 02.30.Nw, 05.20.Dd; secondary 02.30.Uu, 43.20.+g, 47.10.-g, 47.10.ab.

Keywords: non-locality, thermo-viscoelastic waves, bivelocity fluid mechanics, Maxwell-Cattaneo relaxation, Chapman-Enskog kinetic theory. 
from strong inhomogenieties due to large temperature or density gradients. This ostensibly motivates his revised constitutive theory, "bivelocity fluid mechanics", based on the notion that barycentric velocity, associated with material inertia and kinetic energy, is not appropriate for the description of internal stress in a fluid or solid. In its place, he proposes a "volume" or "work" velocity, together with various constitutive models for the "diffuse volume flux" representing the difference between the two velocities. This stratagem serves inter alia to introduce higher spatial gradients of temperature and velocity into the constitutive theory.

An alternative perspective is offered in [G10], where it is argued that the above revision is necessitated by the breakdown of the thermo-mechanically simple material of Coleman $[1961 ; 1964]$ and that Brenner's constitutive theory is a restricted version of a more general non-local theory. Such a theory, anticipated in numerous previous works (see [Eringen 2002] and references therein), was sketched out in [G10], which leaves unanswered certain questions regarding the magnitude of material-specific length and time scales involved in the breakdown of the classical model and the inadequacy of the bi-velocity model as a strictly linear theory.

The purpose of the present work is to elucidate further the above questions, by considering specific models that are fully non-local in both space and time, i.e., models which involve long-range interactions in space combined with long-range history effects in time. In particular, we show that the linear model which emerges at "Burnett order" in the classical Chapman-Enskog kinetic theory is a special case of the general model. As appreciated by others [Müller and Ruggeri 1998], this kinetic theory involves relaxation effects of the type described earlier by Maxwell's viscoelasticity [1867] and later by Cattaneo's retarded thermal conductivity [1948]. As we shall also show, Brenner's theory represents a restriction to the dissipative response arising on time scales longer that the Maxwell-Cattaneo relaxation times. Also, it is shown that a fully non-local model can be reconstructed from the linearized Chapman-Enskog theory.

Acoustic wave propagation represents a plausible testing ground for non-local thermo-mechanical effects, as already recognized in [Davis and Brenner 2012]; the present paper provides an extension of that work. It also presents an extension of [G10] that identifies the hyperstresses conjugate to higher velocity gradients. However, the applications to wave propagation are restricted to the linear momentum balance, with no account taken of higher-order inertial terms. Finally, we establish a connection to various non-local models of wave propagation in complex solids.

\section{Fourier-Laplace representation: Recapitulation of previous work}

Following the analysis of [G10], we recall that Fourier representations embody the notion of wave-number dependent transport coefficients, capturing the dispersive 
effects associated with higher gradients. When extended to the time domain by means of the Laplace transform, one obtains a similar description of frequency effects in materials with memory ${ }^{1}$. Hence, the transform

$$
\hat{\boldsymbol{\psi}}(\boldsymbol{k}, s)=\hat{\boldsymbol{\psi}}_{t}(\boldsymbol{k}, s)=\frac{1}{\sqrt{8 \pi^{3}}} \int_{\mathscr{R}} \int_{0}^{\infty} e^{-\imath \boldsymbol{k} \cdot \boldsymbol{x}-s t^{\prime}} \boldsymbol{\psi}\left(\boldsymbol{x}, t-t^{\prime}\right) \mathrm{d} V(\boldsymbol{x}) \mathrm{d} t^{\prime}
$$

provides a localized description in Fourier space $(\boldsymbol{k}, s)$ of a spatio-temporally delocalized field in physcial space, $\psi_{t}\left(\boldsymbol{x}^{\prime}, t^{\prime}\right)=\boldsymbol{\psi}\left(\boldsymbol{x}^{\prime}, t-t^{\prime}\right), \quad \boldsymbol{x}^{\prime} \in \mathscr{R}, \quad t^{\prime} \geq 0$, and vice versa. Accordingly, a causal, non-local and linear constitutive equation between two sets of tensor fields

$$
\boldsymbol{\Phi}(\boldsymbol{x}, t)=\left\{\boldsymbol{\varphi}^{(1)}, \ldots, \boldsymbol{\varphi}^{(m)}\right\}(\boldsymbol{x}, t) \quad \text { and } \quad \boldsymbol{\Psi}(\boldsymbol{x}, t)=\left\{\boldsymbol{\psi}^{(1)}, \ldots, \boldsymbol{\psi}^{(m)}\right\}\left(\boldsymbol{x}, t-t^{\prime}\right),
$$

for $t^{\prime} \geq 0$, of the type pursued by Eringen [1992; 2002], can be represented by the linear form:

$$
\widehat{\boldsymbol{\Phi}}(\boldsymbol{k}, s)=\widehat{\mathfrak{L}}(\boldsymbol{k}, s) \widehat{\boldsymbol{\Psi}}(\boldsymbol{k}, s)
$$

where $\hat{\mathfrak{L}}$ represents a matrix of tensor moduli. This relation is tantamount to the spectral theory of commutative linear operators with $\left\{{ }_{\imath} \boldsymbol{k}, s\right\} \rightarrow\left\{\nabla, \partial_{t}\right\}$, and the time-honored Fourier-Laplace transforms provide a concrete algebraic representation.

With $\widehat{\boldsymbol{\Phi}}(\boldsymbol{x}, t)=\{\hat{\boldsymbol{\sigma}}, \hat{\boldsymbol{q}}\}$ representing stress $\hat{\boldsymbol{\sigma}}$ and heat flux $\hat{\boldsymbol{q}}$ in (2), one obtains a linear non-local theory of thermo-viscoelasticity. We recall that Eringen [2002, Section 7] proposes a simpler non-local theory for viscous incompressible fluids with uncoupled heat flux, a theory that was overlooked in [G10].

If we adopt a scaling in which $\boldsymbol{k}$ and $s$ are replaced by non-dimensional forms $\lambda_{0} \boldsymbol{k}$ and $\tau_{0} s$, with $\lambda_{0}$ and $\tau_{0}$ denoting, respectively, appropriate material length and time scales, then $k=|\boldsymbol{k}|$ and $s$ represent, respectively, a Knudsen and a Deborah number. Hence, one obtains a weakly non-local spatio-temporal models from the Taylor-series expansion of $\hat{\mathfrak{L}}(\boldsymbol{k}, s)$ about the spatially uniform steady state $k=0, s=0$. The expansion in $\boldsymbol{k}$ is, to terms $O\left(k^{2}\right)$, tantamount to the Burnett expansion of kinetic theory [Müller and Ruggeri 1998], whereas the expansion in $s$ represents the "retarded motions" of [Coleman and Noll 1961; Coleman 1964]. In particular, the simple fluid emerges at $O(k)$ in $\boldsymbol{k}$. Dissipative response, defining the Navier-Stokes-Fourier regime, arises for $s \rightarrow 0$ at $O(1)$ for $\hat{\boldsymbol{q}}$ and at $O(s)$ for $\hat{\boldsymbol{\sigma}}$, provided we take $\hat{\boldsymbol{v}}$ to be $O(s)$, i.e.,

$$
\boldsymbol{v}=\partial_{t} \boldsymbol{u}, \quad \text { with } \quad \therefore \quad \hat{\boldsymbol{v}}=s \hat{\boldsymbol{u}},
$$

where $\boldsymbol{u}$ denotes material displacement from the positions at $t=0$.

\footnotetext{
${ }^{1}$ Since our "Fourier-Laplace" transform involves what is essentially a Fourier transform on $t=$ $[0, \infty)$ with complex wave vector $s$, we could as well employ the terminology "Fourier transform" and "Fourier space".
} 
Following [G10]we consider a spatially nonlocal linear viscoelastic fluid in which the stress $\sigma$ relative to a spatially uniform equilibrium pressure $p_{0}$ (replacing the deviatoric stress $\tau$ of [G10], which deals with incompressible fluids) and the heat flux $\boldsymbol{q}$, are represented by $\hat{\boldsymbol{\sigma}}\left[=\hat{\sigma}_{i j}\right]$ and $\hat{\boldsymbol{q}}\left[=\hat{q}_{i}\right]$ as functions of velocity $\hat{\boldsymbol{v}}\left[=\hat{v}_{i}\right]$ and departure $\hat{\theta}=\hat{T}-T_{0} / s$ from the uniform absolute temperature $T_{0}$ of the equilibrium base state. With Cartesian tensor components displayed for clarity, these can be written as in [G10]:

$$
\begin{aligned}
\hat{\boldsymbol{q}}\left[=\hat{q}_{i}\right]=\hat{\boldsymbol{L}}^{(11)} \hat{\theta}+\hat{\boldsymbol{L}}^{(12)} \hat{\boldsymbol{v}} & {\left[\stackrel{\text { def }}{=} \hat{L}^{(11)} \hat{\theta}+\hat{L}_{i j}^{(12)} \hat{v}_{j}\right], } \\
\hat{\boldsymbol{\sigma}}\left[\hat{\sigma}_{i j}\right]=\hat{\boldsymbol{L}}^{(21)} \hat{\theta}+\hat{\boldsymbol{L}}^{(22)} \hat{\boldsymbol{v}} & {\left[\stackrel{\text { def }}{=} \hat{L}_{i j}^{(21)} \hat{\theta}+\hat{L}_{i j l}^{(22)} \hat{v}_{l}\right], }
\end{aligned}
$$

where the tensor coefficients $\hat{L}$ depend on the complex frequency $s$ and wave vector $\boldsymbol{k}$. Here as in the following, we indicate components of tensors on a given Cartesian system by means of square brackets [ ], and the Cartesian summation convention is employed. We further employ colons to denote contraction of the trailing components of a prefactor with all the components of the postfactor, with the conventional dot for the scalar product of vectors.

For isotropic materials, the various tensors in (5) must be isotropic functions of the wave vector and, for the case of symmetric stress assumed here, can be written down explicitly as in [G10]:

$$
\begin{aligned}
& \hat{L}_{i}^{(11)}=\hat{A} k_{i}, \\
& \hat{L}_{i j}^{(12)}=\hat{B} \delta_{i j}+\hat{C} k_{i} k_{j}, \\
& \hat{L}_{i j}^{(21)}=\hat{D} \delta_{i j}+\hat{E} k_{i} k_{j}, \\
& \hat{L}_{i j l}^{(22)}=\hat{F} \delta_{i j} k_{l}+\hat{G}\left(\delta_{i l} k_{j}+\delta_{j l} k_{i}\right)+\hat{H} k_{i} k_{j} k_{l},
\end{aligned}
$$

where the scalar coefficients $\hat{A}, \hat{B}, \ldots, \hat{H}$ are functions of $s$ and $k^{2}$, where $k^{2}=k_{i} k_{i}$ defines a generally complex quantity, since we shall admit complex wave vectors $\boldsymbol{k}$. Also, we have added carats to the coefficients defined in [G10], in order to distinguish them from their physical-space images considered below.

In direct tensor notation, the preceding relations become

$$
\begin{aligned}
& \hat{\boldsymbol{q}}=\hat{A} \boldsymbol{k} \hat{\theta}+(\hat{B} \mathbf{1}+\hat{C} \boldsymbol{k} \otimes \boldsymbol{k}) \hat{\boldsymbol{v}}, \\
& \hat{\boldsymbol{\sigma}}=(\hat{D} \mathbf{1}+\hat{E} \boldsymbol{k} \otimes \boldsymbol{k}) \hat{\theta}+\hat{F}(\boldsymbol{k} \cdot \hat{\boldsymbol{v}}) \mathbf{1}+\hat{G}(\boldsymbol{k} \otimes \hat{\boldsymbol{v}}+\hat{\boldsymbol{v}} \otimes \boldsymbol{k})+\hat{H}(\boldsymbol{k} \cdot \hat{\boldsymbol{v}}) \boldsymbol{k} \otimes \boldsymbol{k} .
\end{aligned}
$$

Now, the requirement of real $\boldsymbol{q}$ and $\boldsymbol{\sigma}$ implies that the coefficient, say, $\hat{K}_{n}$ of the general term in (7):

$$
\hat{K}_{n}(k, s) \boldsymbol{k}^{n}, \quad \text { where } \quad \boldsymbol{k}^{n}=\boldsymbol{k}^{n-1} \otimes \boldsymbol{k}, \quad n=1,2, \ldots, \quad \boldsymbol{k}^{0}=1,
$$


must satisfy $\hat{K}_{n}^{*}\left(k^{2}, s\right)=(-1)^{n} \hat{K}_{n}\left(k^{* 2}, s\right)$, where asterisks denote complex conjugates here and below. Hence, the coefficients of even (odd) order terms in $\boldsymbol{k}$ must be essentially real (imaginary). (By essentially real we mean a function $\mathbb{R} \rightarrow \mathbb{R}$, i.e., a function that is real-valued when its arguments are real, whereas essentially imaginary is a function $\mathbb{R} \rightarrow \imath \mathbb{R}$, i.e., $\imath$ times an essentially real function.)

In line with the above remarks, and following [G10], we obtain from (6) a weakly nonlocal theory in space by means of the wave-number expansions of $\hat{K}=\hat{A}, \hat{B}, \ldots, \hat{H}$ of the form

$$
\hat{K}=\hat{K}_{0}(s)+\hat{K}_{1}(s) k^{2}+\hat{K}_{2}(s) k^{4}+\cdots,
$$

where $\hat{K}_{m}(s)$ are independent of $\boldsymbol{k}$. As pointed out in [G10], Gallilean invariance of heat flux requires that $\hat{B}_{0}=0$ which, by a general form of Onsager symmetry, implies that $\hat{D}_{0}=0$. We shall show presently that the latter result arises from a properly restricted form of that symmetry.

Note that the stress defined by $(7)_{2}$ represents a non-local quantity whose expansion in $\boldsymbol{k}$ defines a hierarchy of hyperstresses. In particularly, by an extension of the dissipative forms discussed by [Goddard and Lee 2017] we have

$$
\hat{\boldsymbol{\sigma}}=\sum_{m \geq 1} \hat{\boldsymbol{\sigma}}^{(m)}:(-\imath \boldsymbol{k})^{m-1}, \quad \text { i.e., } \quad \hat{\sigma}_{i j}=\sum_{m \geq 1} \hat{\sigma}_{i j, j_{1}, \ldots, j_{m-1}}^{(m)}(-\imath \boldsymbol{k})_{j_{1}, \ldots, j_{m-1}}^{m-1},
$$

where $\hat{\boldsymbol{\sigma}}^{(1)}$ is Cauchy stress and the $\hat{\boldsymbol{\sigma}}^{(m)}, \quad m>1$, is the hyperstress conjugate to $(\imath \boldsymbol{k})^{m} \hat{\boldsymbol{v}}$.

Now, if both $\hat{B}_{0}$ and $\hat{D}_{0}$ vanish, then (5) and (7) reduce to a standard form in which $\{\nabla \theta, \operatorname{Sym}(\nabla \boldsymbol{v})\}$ represent nine forces conjugate to nine fluxes $\{\boldsymbol{q}, \boldsymbol{\sigma}\}$. In that case, the local dissipation rate is given by:

$$
\boldsymbol{\sigma}: \nabla \boldsymbol{v}-\frac{\boldsymbol{q}}{T_{0}} \cdot \nabla \theta \geq 0
$$

in the dissipative regime, where $\sigma, \boldsymbol{q}$ are strictly dissipative. Thus, by the ParsevalPlancherel theorem, the global dissipation becomes ${ }^{2}$

$$
\begin{aligned}
\int_{\Re}(\boldsymbol{\sigma} & \left.: \nabla \boldsymbol{v}-\frac{\boldsymbol{q}}{T_{0}} \cdot \nabla \theta\right) \mathrm{d} V(\boldsymbol{x}) \\
& =-\imath \int_{\hat{\mathscr{R}}}\left(\hat{\boldsymbol{\sigma}}: \boldsymbol{k}^{*} \hat{\boldsymbol{v}}^{*}-\hat{\boldsymbol{q}} \cdot \boldsymbol{k}^{*} \frac{\hat{\theta}^{*}}{T_{0}}\right) \mathrm{d} \hat{V}(\boldsymbol{k}) \\
& =-\imath \int_{\hat{\mathscr{R}}}\left(\hat{L}_{i j}^{(21)} k_{i}^{*} \hat{v}_{j}^{*} \frac{\hat{\theta}}{T_{0}}+\hat{L}_{i j l}^{(22)} k_{i}^{*} \hat{v}_{j}^{*} \hat{v}_{l}-\hat{L}_{i}^{(11)} k_{i}^{*} \frac{|\hat{\theta}|^{2}}{T_{0}^{2}}-\hat{L}_{i j}^{(12)} k_{i}^{*} \hat{v}_{j} \frac{\hat{\theta}^{*}}{T_{0}}\right) \mathrm{d} \hat{V}(\boldsymbol{k}) \\
& \geq 0,
\end{aligned}
$$

\footnotetext{
${ }^{2}$ after extension to complex $\boldsymbol{k}$ and correction of a typographical error of [G10]
} 
where $\mathscr{R}$ is the spatial region occupied by the fluid and $\hat{\mathscr{R}}$ is its Fourier image (i.e., the transform of its indicator function). Based on an unwarranted restriction to real-valued transforms $\hat{\theta}, \hat{\boldsymbol{v}}$, it is erroneously concluded in [G10] that the general Onsager symmetry $L_{i j}^{(21)}=L_{i j}^{(12)}$ eliminates dissipative coupling between temperature and velocity.

As a more restricted form of Onsager symmetry, note that (7) gives

$$
\begin{aligned}
& \hat{p}=\hat{I} \hat{\theta}+\hat{J} \boldsymbol{k} \cdot \hat{\boldsymbol{v}}, \quad \text { where } \hat{I}=-\left(\hat{D}+\hat{E} k^{2} / 3\right), \hat{J}=-\left(\hat{F}+2 \hat{G} / 3+\hat{H} k^{2} / 3\right), \\
& \boldsymbol{k} \cdot \hat{\boldsymbol{q}}=\hat{A} k^{2} \hat{\theta}+\left(\hat{B}+\hat{C} k^{2}\right) \boldsymbol{k} \cdot \hat{\boldsymbol{v}},
\end{aligned}
$$

with $J \rightarrow J_{0}=-\imath \beta_{0}$ for $k \rightarrow 0$, where $\beta_{0}$ denotes the standard bulk (or "volume") viscosity. Thus, in the dissipative regime, the quantities

$$
\theta \nabla \cdot \boldsymbol{q} / T_{0}-p \nabla \cdot \boldsymbol{v} \geq 0, \quad \text { or } \quad l\left(\hat{\theta}^{*} \boldsymbol{k} \cdot \hat{\boldsymbol{q}} / T_{0}-\hat{p}^{*} \boldsymbol{k} \cdot \hat{\boldsymbol{v}}\right) \geq 0
$$

represent the dissipation rate. The significance of the term involving pressure work is obvious, while the other term is essentially the potential Carnot work dissipated locally by irreversible heat flow, since $\theta / T_{0}=-\left(1-T / T_{0}\right)$. Hence, the Onsager symmetry of the linear relations (12) requires that

$$
\left(\hat{B}+\hat{C} k^{2}\right) / T_{0}=-\hat{I}=\left(\hat{D}+\hat{E} k^{2} / 3\right), \quad \text { and } \quad \therefore \quad \hat{D}_{0}=\hat{B}_{0}=0
$$

in the dissipative regime, a necessary restriction on the more general form proposed in [G10].

As they stand, the relations (7) represent linear non-local thermo-viscoelasticity, with the $x$ - $t$ images of the coefficients $\hat{K}=\hat{A}, \hat{B}, \hat{C}, \ldots$ providing the kernels of integral operators acting on $\mathfrak{f}=\{\theta, \boldsymbol{v}\}$. According to (8) these assume the form

$$
\hat{K}_{n} \boldsymbol{k}^{n}(\cdot) \hat{\mathfrak{f}} \rightarrow(-\imath)^{n} \int_{t^{\prime}=0}^{\infty} \int_{\mathscr{R}^{\prime}} K_{n}\left(\boldsymbol{x}^{\prime}, t^{\prime}\right) \nabla^{n}(\cdot) \mathfrak{f}\left(\boldsymbol{x}-\boldsymbol{x}^{\prime}, t-t^{\prime}\right) \mathrm{d} V\left(\boldsymbol{x}^{\prime}\right) \mathrm{d} t^{\prime},
$$

where $(\cdot)$ represents an optional dot product or contraction. Moreover, since the coefficients $\hat{K}$ are functions of $\boldsymbol{k}$ that depend only on $k^{2}$, they admit simplified inverse spatial transforms, as discussed in the Appendix. We now consider the special cases of the general theory represented by the kinetic theory of gases and by Brenner's bivelocity model.

\section{Linearized kinetic theory of gases}

As a slight variant on the kinetic-theory results given by Chapman and Cowling [1960, p. 410], Müller and Ruggeri [1998, p. 74] give the following implicit forms 
for heat flux and shear stress in a monatomic gas:

$$
\begin{array}{r}
\boldsymbol{q}=-\tau_{q}\left\{-\frac{5}{2} R p \nabla \theta+\dot{\boldsymbol{q}}+\boldsymbol{q} \cdot \nabla \boldsymbol{v}-R \theta \nabla \cdot \boldsymbol{\sigma}-\frac{7}{5} \boldsymbol{q} \nabla \cdot \boldsymbol{v}-\frac{4}{5} \boldsymbol{q} \cdot \nabla \boldsymbol{v}+\frac{7}{2} R \boldsymbol{\sigma} \cdot \nabla \theta+\frac{\boldsymbol{\sigma}}{\rho} \cdot \nabla p\right\}, \\
\boldsymbol{\sigma}=\tau_{\sigma}\left\{p\left[\nabla \boldsymbol{v}+(\nabla \boldsymbol{v})^{T}-\frac{2}{3} \nabla \cdot \boldsymbol{v} \mathbf{1}\right]-\dot{\boldsymbol{\sigma}}-2\left[\boldsymbol{\sigma} \nabla \boldsymbol{v}+(\boldsymbol{\sigma} \nabla \boldsymbol{v})^{T}\right]\right. \\
\left.+\frac{2}{5}\left[\nabla \boldsymbol{q}+(\nabla \boldsymbol{q})^{T}-\frac{2}{3} \nabla \cdot \boldsymbol{q} \mathbf{1}\right]-\boldsymbol{\sigma} \nabla \cdot \boldsymbol{v}\right\},
\end{array}
$$

where $\tau_{\sigma}$ and $\tau_{q}$ are the respective relaxation times for stress and heat flux, and $R$ is the species-specific gas constant in the ideal-gas law $R=\rho T / p$. By means of the the leading linear terms in (15), we identify the Newtonian shear viscosity and the Fourier conductivity, respectively, as

$$
\begin{aligned}
& \mu=p \tau_{\sigma}, \\
& \kappa=5 \operatorname{Rp} \tau_{q} / 2=5\left(\tau_{q} / \tau_{\sigma}\right) R \mu / 2 .
\end{aligned}
$$

Taking $\tau_{q}=3 \tau_{\sigma} / 2$ one recovers a standard approximation $\kappa \doteq 5 c_{V} \mu / 2$ for smooth spherically-symmetric molecules [Chapman and Cowling 1960, p. 273] with specific heat $c_{V}=3 R / 2$.

It is a straighhtforward matter to linearize the equations (15) about a uniform state of density $\rho_{0}$, temperature $T_{0}$ and pressure $p_{0}=p_{\text {eq }}\left(\rho_{0}, T_{0}\right)$, since terms involving products of quantities that vanish in the uniform state do not contribute to the linearized equations. The function $p_{\text {eq }}$ introduced here represents the equilibrium equation of state, which is of course given by the above ideal-gas law for dilute gases, but we allow here a more general equation of state.

Letting

$$
\begin{array}{ll}
\mu=\rho_{0} v=\frac{\mu_{0}}{\left(1+\tau_{\sigma 0} s\right)}, & \tau=\frac{4 \tau_{\sigma 0}}{5\left(1+\tau_{\sigma 0} s\right)}, \\
\kappa=\rho_{0} c_{p 0} \alpha=\frac{\kappa_{0}}{\left(1+\tau_{q 0} s\right)}, & f_{0}=\frac{2 T_{0}}{5 p_{0}},
\end{array}
$$

one finds that the linearized equations take on this compact form in Fourier space:

$$
\begin{aligned}
\hat{\boldsymbol{\sigma}} & =2 \mu \hat{\boldsymbol{\epsilon}}+\imath \tau[(\boldsymbol{k} \otimes \hat{\boldsymbol{q}}+\hat{\boldsymbol{q}} \otimes \boldsymbol{k}) / 2-(\hat{\boldsymbol{q}} \cdot \boldsymbol{k}) \mathbf{1} / 3] \\
\text { and } \quad \hat{\boldsymbol{q}} & =-\imath \kappa \boldsymbol{k} \hat{\theta}+\imath \kappa f_{0} \boldsymbol{k} \cdot \hat{\boldsymbol{\sigma}}, \\
\text { where } \quad \hat{\boldsymbol{\epsilon}} & =\imath[(\boldsymbol{k} \otimes \hat{\boldsymbol{v}}+\hat{\boldsymbol{v}} \otimes \boldsymbol{k}) / 2-(\boldsymbol{k} \cdot \hat{\boldsymbol{v}}) \mathbf{1} / 3] .
\end{aligned}
$$

After a bit of algebra, one can solve equations (18) for $\hat{\boldsymbol{q}}, \hat{\boldsymbol{\sigma}}$ in terms of $\hat{\theta}, \hat{\boldsymbol{v}}$, to give

$\hat{\boldsymbol{q}}=-\kappa\left\{\imath \boldsymbol{k} \hat{\theta} f_{1}+\mu f_{0} f_{2}\left[k^{2} \hat{\boldsymbol{v}}+f_{1} \boldsymbol{k} \otimes \boldsymbol{k} \cdot \hat{\boldsymbol{v}} / 2\right]\right\}$,

$\hat{\boldsymbol{\sigma}}=2 \imath \mu f_{2}[(\boldsymbol{k} \otimes \hat{\boldsymbol{v}}+\hat{\boldsymbol{v}} \otimes \boldsymbol{k}) / 2-(\boldsymbol{k} \cdot \hat{\boldsymbol{v}}) \mathbf{1} / 3]+\kappa \tau f_{1}\left(\boldsymbol{k} \otimes \boldsymbol{k}-k^{2} \mathbf{1} / 3\right)\left[\hat{\theta}-\imath \mu f_{0} f_{2}(\boldsymbol{k} \cdot \hat{\boldsymbol{v}}) / 3\right]$,

where $f_{1}=\left[1+2(\lambda k)^{2} / 3\right]^{-1}, \quad f_{2}=\left[1+(\lambda k)^{2} / 2\right]^{-1}, \quad \lambda=\sqrt{\kappa \tau f_{0}}$. 
The terms in $\hat{\theta}$ appearing in the expression for $\hat{\boldsymbol{\sigma}}$ represent a non-local form of Maxwell's celebrated thermal stress [Maxwell 1879, Eqs. (53)-(54)] in a rarefied gas. According to Maxwell's kinetic theory, a good estimate of the magnitude of this stress relative to the Newtonian viscous stress is $v_{0}\left|\nabla^{2} \theta\right| / T_{0} \sqrt{ } \operatorname{tr}\left(\epsilon^{2}\right)$, where $v_{0}=\mu_{0} / \rho_{0}$ is the kinematic viscosity and $\sqrt{ } \operatorname{tr}\left(\epsilon^{2}\right)$ the effective shear rate. Hence, according to the kinetic theory, the thermal stress will generally to be important only in the slow shearing of a rarified gas, as pointed out by Maxwell and noted in [G10].

Comparing with the general forms (7), one finds that the coefficients $\hat{A}, \hat{B}, \ldots, \hat{H}$ are given by

$$
\begin{array}{lll}
\hat{A}=-\imath \kappa f_{1}, & \hat{B}=-\kappa \mu k^{2} f_{0} f_{2}, & \hat{C}=-\kappa \mu f_{0} f_{1} f_{2} / 3, \\
\hat{E}=\kappa \tau f_{1}, & \hat{G}=\imath \mu f_{2}, & \hat{H}=-\imath \kappa \tau \mu f_{0} f_{1} f_{2} / 3,
\end{array}
$$

and it is easy to obtain expansions in $k^{2}$ of the type (9).

According to the kinetic theory of dilute monatomic gases, the irreversible contribution to pressure vanishes [Chapman and Cowling 1960; Müller and Ruggeri 1998], implying that the coefficients $\hat{I}, \hat{K}$ in (12) are zero and hence that

$$
\hat{D}=-\hat{E} k^{2} / 3 \text { and } \hat{F}=-2 \hat{G} / 3-\hat{H} k^{2} / 3 \text {, }
$$

determining the remaining coefficients $\hat{D}, \hat{F}$. However, one should not expect these relations to hold for more general fluids, such as liquids and polyatomic gases, whose bulk viscosity $\beta_{0}=\imath \hat{J}_{0}=-l\left(\hat{F}_{0}+2 / 3 \hat{G}_{0}\right)$ is generally non-zero.

The terms in $(17)$ of the form $(1+\tau s)$ represent exponential relaxation in the time domain. As such, they describe Maxwell's viscoelasticity and Cataneo's heat conduction, which admit both mechanical shear waves and heat waves, reflecting a breakdown of purely diffusive, dissipative response on time scales $\tau$. We recall that Ignaczak and Ostoja-Starzewski [2009] give a comprehensive treatment of the local theory of finite thermoelastic wave speeds, represented by terms $O(k)$ in (19). By contrast, and as anticipated above, we expect dissipative response to arise in the small Deborah number limit $D e=\tau_{0} s \ll 1$.

It is shown in the Appendix that one can analytically determine the inverse transforms of the coefficients in (20) by means of the formula (42), thereby providing the kernels in the integral operator (14). This provides a fully non-local model which should be much superior to weakly non-local models involving a sequence of higher spatial gradients, since integral operators, in contrast to differential operators, are generally bounded. This is especially significant in the neighborhood of singularities, as illustrated by the well-known work of Eringen [2002, Section 6.14] on crack-tip stresses in linear elasticity. 


\section{Bivelocity model}

Here, we analyze here a recent version of Brenner's bi-velocity model [2009], in order to compare it with the linear theory proposed above. Given that Brenner's modeling rests heavily on appeals to linear irreversible thermodynamics ("LIT"), it is appropriate to employ a fully linearized version of the type employed in the present paper and, also, to restrict the analysis to dissipative response.

Since Brenner employs somewhat special variables and notation, we have included Table 1 below to clarify the relation of his variables to those employed in the present work.

We adopt that form of Brenner's model which he deems appropriate to creeping (inertialess) flow ${ }^{4}$, as represented by Eqs. (2.7), (2.12) and (2.13) of [Brenner 2009]. In the present notation, these become

$\boldsymbol{q}=-\kappa_{0} \nabla \theta+L_{12} \nabla p_{e q}-p_{e q} \boldsymbol{j}_{w} \doteq-\kappa_{0} \nabla \theta+L_{12}\left[\left(\partial_{\theta} p\right)_{0} \nabla \theta+\left(\partial_{\rho} p\right)_{0} \nabla \rho\right]-p_{0} \boldsymbol{j}_{w}$

$\boldsymbol{j}_{w}=-L_{21} T^{-1} \nabla \theta+L_{22} \nabla p_{e q} \doteq-L_{21} T_{0}^{-1} \nabla \theta+L_{22}\left[\left(\partial_{\theta} p\right)_{0} \nabla \theta+\left(\partial_{\rho} p\right)_{0} \nabla \rho\right]$

$\boldsymbol{\sigma}=2 \mu_{0} \overline{\nabla \boldsymbol{v}}_{w}=2 \mu_{0}\left[\overline{\nabla \boldsymbol{v}}+\bar{\nabla}_{w}\right], \quad p=-\beta_{0} \nabla \cdot \boldsymbol{v}_{w}=-\beta_{0} \nabla \cdot\left(\boldsymbol{v}+\boldsymbol{j}_{w}\right)$,

where overbars represent symmetric deviators and $\doteq$ denotes the approximation arising from linearization about the uniform base state employed elsewhere in the present article. In Brenner's model, the coefficients $L_{i j}$ are assumed to describe a dissipative linear system, with corresponding Onsager symmetry $L_{21}=L_{12}$.

\footnotetext{
${ }^{4}$ Otherwise, his constitutive equations appear to contain inertial terms that are hard to reconcile with the principle of material frame indifference.
}

\begin{tabular}{l|c|c} 
Quantity & [Brenner 2009] & Present \\
\hline absolute temperature & $T$ & $T_{0}+\theta$ \\
barycentric velocity & $\boldsymbol{v}_{m}$ & $\boldsymbol{v}$ \\
"work"3 or "volume" velocity & $\boldsymbol{v}_{w}$ & $\boldsymbol{v}_{w}$ \\
diffuse "volume" flux & $\boldsymbol{j}_{w}=\boldsymbol{v}_{w}-\boldsymbol{v}_{m}$ & $\boldsymbol{j}_{w}=\boldsymbol{v}_{w}-\boldsymbol{v}$ \\
pressure tensor & $\boldsymbol{P}$ & $p_{e q} \mathbf{1}-\boldsymbol{\sigma}$ \\
pressure & $\bar{p}=\operatorname{tr}(\boldsymbol{P}) / 3$ & $p=p_{e q}-\operatorname{tr}(\boldsymbol{\sigma}) / 3$ \\
"thermodynamic" pressure & $p$ & $p_{e q}$ \\
shear stress & $\boldsymbol{T}$ & $\boldsymbol{\tau}=\boldsymbol{\sigma}+p \boldsymbol{I}$ \\
heat flux & $\boldsymbol{j}_{u}$ & $\boldsymbol{q}$ \\
"entropic" heat flux & $\boldsymbol{q}=\boldsymbol{j}_{u}+p \boldsymbol{j}_{w}$ & $\boldsymbol{q}+p_{e q} \boldsymbol{j}_{w}$ \\
thermal conductivity for $\boldsymbol{q}$ & $k$ & $\kappa_{0}$ \\
shear and bulk viscosity & $\eta, \zeta$ & $\mu_{0}, \beta_{0}$
\end{tabular}

Table 1. Variables and notation. 
To compare with the present constitutive theory, it suffices to eliminate $\boldsymbol{j}_{w}$ from (22), and it is algebraically expedient to express these relations as Fourier-Laplace transforms. Account taken of the linearized mass balance (cf. Eqs. (27) below), one thereby obtains relations of the form (7) and (12), with

$$
\begin{aligned}
& \hat{A}=\imath\left\{\left[L_{12}-p_{0} L_{22}\right]\left(\partial_{\theta} p\right)_{0}-\kappa_{0}+L_{12} p_{0} / T_{0}\right\}, \hat{B}=0, \\
& \hat{C}=\left[L_{12}-p_{0} L_{22}\right] \rho_{0}\left(\partial_{\rho} p\right)_{0} / s, \quad \hat{E}=-2 \mu_{0}\left[L_{22}\left(\partial_{\theta} p\right)_{0}-L_{12} / T_{0}\right], \\
& \hat{G}=\imath \mu_{0}, \quad \hat{H}=2 \imath \mu_{0} \rho_{0}\left(\partial_{\rho} p\right)_{0} L_{22} / s, \\
& \hat{I}=\beta_{0}\left[L_{22}\left(\partial_{\theta} p\right)_{0}-L_{12} / T_{0}\right] k^{2}=-\left(\hat{D}+\hat{E} k^{2} / 3\right), \\
& \hat{J}=-\imath \beta_{0}\left[1+\rho_{0}\left(\partial_{\rho} p\right)_{0} L_{22} k^{2} / s\right]=-\left(\hat{F}+2 \hat{G} / 3+\hat{H} k^{2} / 3\right),
\end{aligned}
$$

from which it follows that

$$
\beta_{0}=2 \mu_{0} / 3, \quad \hat{D}=0 . \quad \hat{F}=0
$$

Note that [Brenner 2009] takes

$L_{12}=T_{0} \alpha_{0} \beta_{0}, \quad$ where $\quad \alpha_{0}=\kappa_{0} / \rho_{0} c_{p 0}, \beta_{0}=-\left(\partial_{\theta} \rho\right)_{p 0} / \rho_{0}=\left(\partial_{\theta} p\right)_{0} / \rho_{0}\left(\partial_{\rho} p\right)_{0}$, involving the thermal diffusivity $\alpha_{0}$ and isobaric coefficient of thermal expansion $\beta_{0}$. With certain reservations, he then takes $L_{22}=\alpha_{0} \beta_{0} /\left(\partial_{\theta} p\right)_{0}$, which would imply that $L_{22}\left(\partial_{\theta} p\right)_{0}-L_{12} / T_{0}$ and, hence, $\hat{I}$ and $\hat{E}$ vanish in (23). Thus, the Maxwell thermal stress represented by the term $\hat{E}$ in (7) also vanishes.

Brenner does not invoke the restrictions on the coefficients of viscosity $\mu_{0}, \beta_{0}$ that are required for consistency with the general model proposed in this work.

\section{Application to linear thermo-acoustic waves}

For the uniform fluid at rest, we adopt mechanical and caloric equations of state connecting equilibrium pressure and specific internal energy to temperature and density:

with

$$
p=p_{\mathrm{eq}}(\theta, \rho) \quad \text { and } \quad \varepsilon=\varepsilon_{\mathrm{eq}}(\theta, \rho)
$$

$$
\partial_{\theta} \varepsilon_{\mathrm{eq}}=c_{v}, \quad \partial_{\rho} \varepsilon_{\mathrm{eq}}=\frac{1}{\rho^{2}}\left[p-\theta\left(\partial_{\theta} p_{\mathrm{eq}}\right)\right],
$$

where $c_{v}$ denotes the isochoric specific heat.

The present treatment of temperature and density as independent variables is inspired by the modern literature on continuum thermodynamics, where various intensive variables are given as derivatives of Helmholtz free energy. It seems to us more natural than the formulation based on pressure and entropy adopted in standard treatises on acoustics [Pierce 1981] but in any case can be easily converted to the latter. Accordingly, we shall refer to the "entropy mode" identified 
by [Pierce 1981, p. 523], and subsequently by [Davis and Brenner 2012], as the "thermal mode", noting that the modal amplitudes are simply related by a constant of proportionality [Pierce 1981, Eq. (10-3.16)] according to the linear theory which follows.

Thus, with subscripts 0,1 referring, respectively to a uniform equilibrium state and a small perturbation on that state, such that $\zeta=\zeta_{0}+\zeta_{1}$, for any variable $\zeta$, the linearized balances of momentum, mass, and energy reduce in the absence of body forces or radiant energy transfer to:

$$
\rho_{0} \partial_{t} \boldsymbol{v}_{1}=-\left(\partial_{\theta} p\right)_{0} \nabla \theta_{1}-\left(\partial_{\rho} p\right)_{0} \nabla \rho_{1}+\nabla \cdot \boldsymbol{\sigma}_{1},
$$

where $\quad\left(\partial_{z} p\right)_{0}=\left.\partial_{z} p_{\mathrm{eq}}\right|_{T_{0}, \rho_{0}}, \quad z=\theta, \rho, \quad \partial_{t} \rho_{1}=-\rho_{0} \nabla \cdot \boldsymbol{v}_{1}$,

$$
\text { and } \rho_{0} c_{v 0} \partial_{t} \theta_{1}=\nabla \cdot \boldsymbol{q}_{1}-T_{0}\left(\partial_{\theta} p\right)_{0} \nabla \cdot \boldsymbol{v}_{1} \text {. }
$$

This stated, we shall now drop the subscript 1 on perturbations, as done implicitly in the preceding discussion, where (7) provides constitutive equations for the perturbed heat flux and stress $\boldsymbol{q}$ and $\boldsymbol{\sigma}$ in terms of $\theta$ and $\rho$.

Other than an assumption of a dissipative regime for small $s$, we shall not consider in detail the restrictions on the constitutive model arising from the entropy balance (the Clausius-Duhem inequality) and the related "extended thermodynamics" [Müller and Ruggeri 1998]. However, we note that if heat flux is neglected from (27) the last two members of (27) yield the condition of constant equilibrium entropy $\eta_{\text {eq }}$ :

$$
\rho_{0} \partial_{t} \eta_{\mathrm{eq}}=\rho_{0} c_{v 0} \partial_{t} \theta / T_{0}-\left(\partial_{\theta} p\right)_{0} \partial_{t} \rho / \rho_{0}=0,
$$

whereas the actual entropy $\eta$ may generally increase owing to thermo-mechanical dissipation.

Modulo inhomogeneous terms arising from initial values of $\theta, \rho, \boldsymbol{v}$, the FourierLaplace transforms of (27) reduce to the linear homogeneous form:

$$
\begin{array}{r}
\rho_{0} s \hat{\boldsymbol{v}}+\imath\left(\partial_{\theta} p\right)_{0} \hat{\theta} \boldsymbol{k}+\frac{\rho_{0}}{s}\left(\partial_{\rho} p\right)_{0} \boldsymbol{k} \boldsymbol{k} \cdot \hat{\boldsymbol{v}}-\imath \hat{\boldsymbol{\sigma}} \boldsymbol{k}=\mathbf{0}, \\
\rho_{0} c_{v 0} \hat{\theta}+\imath \boldsymbol{k} \cdot \hat{\boldsymbol{q}}+\imath T_{0}\left(\partial_{\theta} p\right)_{0} \boldsymbol{k} \cdot \hat{\boldsymbol{v}}=0 .
\end{array}
$$

Substitution of (7) into (29) yields a set of four linear equations in $\hat{\theta}, \hat{\boldsymbol{v}}$. However, these can be reduced to a set of two linear equations in $\hat{\theta}, \nabla \cdot v$ by employing the "divergence" form obtained by taking the dot product of $\boldsymbol{k}$ with the first member of (29). The determinantal equation results then in the dispersion relation for the resultant compressive modes:

$$
\begin{aligned}
& {\left[\hat{D}+\hat{E} k^{2}-\left(\partial_{\theta} p\right)_{0}\right]\left[\hat{B}+\hat{C} k^{2}+T_{0}\left(\partial_{\theta} p\right)_{0}\right] k^{2}} \\
& \quad-\left[\rho_{0} c_{v 0} s+\imath \hat{A} k^{2}\right]\left[\rho_{0} s-\left\{2 \imath \hat{G}+\imath \hat{F}-\rho_{0}\left(\partial_{\rho} p\right)_{0} / s\right\} k^{2}-\imath \hat{H} k^{4}\right]=0 .
\end{aligned}
$$


In the application of this relation to the time-periodic waves with temporal frequency $\omega$ it is understood that $s=-i \omega$ here and below.

In addition to the modes described by (30) there exists a decoupled "vorticity" or shearing mode involving the vorticity $\boldsymbol{w}=\nabla \times \boldsymbol{v}$ [Davis and Brenner 2012; Pierce 1990]. By means of the Fourier representation $\hat{\boldsymbol{w}}=\imath \boldsymbol{k} \times \hat{\boldsymbol{v}}$ and the cross product of $\boldsymbol{k}$ with the first member of (29), one obtains

$$
\left[\rho_{0} s-\imath \hat{G}\left(k^{2}, s\right) k^{2}\right] \hat{\boldsymbol{w}}=\mathbf{0},
$$

which has an immediate interpretation in terms of the inverse $G(\boldsymbol{x}, t)$ of the transform $\hat{G}$. As indicated by the analysis in the Appendix, (31) describes shear waves on time scales $\tau_{\sigma 0}$. By contrast, in the dissipative regime that emerges on longer time scales one obtains strongly damped diffusive modes [Davis and Brenner 2012; Pierce 1990].

In sum, given the Fourier-Laplace inverses $A(\boldsymbol{x}, t), B(\boldsymbol{x}, t), \ldots, H(\boldsymbol{x}, t)$, the relations (30)-(31) provide a fully non-local model of linear signal propagation, including long-range memory effects in time. Clearly, a more restricted form is required for most practical applications. Thus, the retention of terms up to $O\left(k^{2}\right)$ in $(7)$ reduces the (30) to

$$
\begin{aligned}
{\left[\hat{D}_{0}+\left(\hat{D}_{1}+\hat{E}_{0}\right) k^{2}-\left(\partial_{\theta} p\right)_{0}\right]\left[\left(\hat{B}_{1}+\hat{C}_{0}\right) k^{2}+T_{0}\left(\partial_{\theta} p\right)_{0}\right] k^{2} } \\
-\left[\rho_{0} c_{v 0} s+\imath \hat{A}_{0} k^{2}\right]\left[\rho_{0} s-\left\{2 \imath \hat{G}_{0}+\imath \hat{F}_{0}-\rho_{0}\left(\partial_{\rho} p\right)_{0} / s\right\} k^{2}\right]=0
\end{aligned}
$$

which involves the five distinct coefficients $\hat{A}_{0}, \hat{B}_{1}+\hat{C}_{0}, \hat{D}_{0}, \hat{D}_{1}+\hat{E}_{0}$ and $\hat{F}_{0}+2 \hat{G}_{0}$, with dependence on $s$ representing relaxation effects in the time domain.

By a slight extension of the kinetic theory of Section 3, four of the coefficients appearing in (32) and the coefficient appearing in the limiting form of (31),

$$
\left[\rho_{0} s-\imath \hat{G}_{0}(s) k^{2}\right] \hat{\boldsymbol{w}}=\mathbf{0},
$$

are given respectively by

$$
\begin{array}{ll}
\imath \hat{A}_{0}=\frac{\kappa_{0}}{1+\tau_{q 0} s}, & \hat{B}_{1}+\hat{C}_{0}=-\frac{4 \kappa_{0} \mu_{0} f_{0}}{3\left(1+\tau_{\sigma 0} s\right)\left(1+\tau_{q 0} s\right)} \\
\hat{D}_{1}+\hat{E}_{0}=\frac{8 \kappa_{0} \tau_{\sigma 0}}{5\left(1+\tau_{q 0} s\right)}, & \iota \hat{F}_{0}+2 \hat{G}_{0}=\frac{\beta_{0}+4 \mu_{0} / 3}{\left(1+\tau_{\sigma 0} s\right)}, \quad \hat{G}_{0}=\frac{\imath \mu_{0}}{1+\tau_{\sigma 0} s}
\end{array}
$$

Note that the form of $\hat{G}_{0}$ and (33) imply elastic shear waves in at high frequencies $s \rightarrow \infty$, thereby eliminating infinite propagation speeds associated with the dissipative limit $s \rightarrow 0$ [Davis and Brenner 2012]. 
Following [Brenner 2009] and [Davis and Brenner 2012], we have included a bulk viscosity coefficient $\beta_{0}$, but which now involves elastic relaxation ${ }^{5}$. The coefficient $\beta_{0}$ vanishes according to the monatomic kinetic theory, as does the remaining unspecified coefficient $\hat{D}_{0}(s)$. Otherwise, we note from (7) that $\hat{D}_{0}(s)$ involves a non-equilibrium response of pressure to temperature variation. We further note that a similar relaxation effect in the temperature-energy response would be obtained upon replacing the specific heat $c_{v 0}$ by an $s$-dependent term $\hat{c}_{v 0}(s)$, analogous to the formalism proposed in [Goddard 1992].

In the dissipative model obtained by neglecting terms $\tau s$ and taking $\hat{D}_{0}=0$ (by the Onsager symmetry discussed above), the dispersion relation (34) reduces to a cubic in both $s$ and $k^{2}$, whereas the model considered by [Davis and Brenner 2012] is cubic in $s$ but quadratic in $k^{2}$.

For the classical Navier-Stokes-Fourier model we have

$$
\begin{array}{ll}
\hat{A}=\hat{A}_{0}=-\imath \kappa_{0}, & \hat{B}=\hat{C}=\hat{D}=\hat{H}=0, \\
\hat{G}=\hat{G}_{0}=\imath \mu_{0}, & \hat{F}_{0}+2 \hat{G}_{0}=-\imath\left(\beta_{0}+4 \mu_{0} / 3\right),
\end{array}
$$

and the dispersion relation (32) reduces to

$$
\begin{gathered}
{\left[s+\alpha_{0} \gamma k^{2}\right]\left[s^{2}-\left(\beta_{0}+4 \mu_{0} / 3\right) s k^{2} / \rho_{0}+\left(\partial_{\rho} p\right)_{0} k^{2}\right]-(\gamma-1) k^{2} s=0, \quad \text { where }} \\
\alpha_{0}=\kappa_{0} / \rho_{0} c_{p 0} \quad \text { and } \quad \gamma=c_{p 0} / c_{v 0}=c_{S}^{2} / c_{T}^{2}=1+T_{0}\left(\partial_{\theta} p\right)_{0}^{2} / \rho_{0}^{2} c_{v 0}\left(\partial_{\rho} p\right)_{0},
\end{gathered}
$$

with $c_{S}$ and $c_{T}$ denoting, respectively, the isentropic and isothermal speeds of sound, whose ratio is given by the specific heat ratio $\gamma$. It is easy to show that (36) is identical with the form given in [Davis and Brenner 2012, Eq. (11)] if $\left(\beta_{0}+4 \mu_{0} / 3\right) / \rho_{0}$ is replaced by the equivalent quantity $\left(2 v_{0}+\lambda_{0}\right)$ in their analysis and $-\rho_{0}^{2}\left(\partial_{\rho} p\right)_{0}$ is identified as the isothermal compressibility.

The more general versions (32) and (34) can be written in the non-dimensional form as

$$
\begin{aligned}
& \left(a \tilde{k}^{2}+b\right)\left(c \tilde{k}^{2}+d\right) \tilde{k}^{2} \tilde{s}-\left(\tilde{s}+e \tilde{k}^{2}\right)\left(\tilde{s}^{2}+f \tilde{k^{2}} \tilde{s}+g \tilde{k}^{2}\right)=0, \\
& \text { where } \quad \tilde{k}^{2}=\tau_{\sigma 0} v_{0} k^{2}, \quad \tilde{s}=\tau_{\sigma 0} s, \\
& \text { with } \quad a=8 \gamma \alpha / 5 \nu_{0}, \quad b=-\left(\partial_{\theta} p\right)_{0} / \rho_{0} c_{v 0}, \\
& \qquad \begin{aligned}
c & =-8 \alpha \nu \rho_{0} c_{p 0} T_{0} / 15 v_{0}^{2} p_{0}, \quad d=T_{0}\left(\partial_{\theta} p\right)_{0} \tau_{\sigma 0} / \mu_{0}, \quad e=\gamma \alpha / v_{0}, \\
f & =-(\beta+4 \mu / 3) / \mu_{0}, \quad g=\left(\partial_{\rho} p\right)_{0} \tau_{\sigma 0} / \nu_{0}=c_{T}^{2} \tau_{\sigma 0} / \nu_{0} .
\end{aligned}
\end{aligned}
$$

Note that $\tilde{k}^{2}$ involves a squared length $\tilde{\lambda}_{0}^{2}=\tau_{\sigma 0} \nu_{0}$, which is related by a factor $|c|$ to that introduced in the Appendix. Note also that for dilute gases all the coefficients $a, b, \ldots, g$ are of order unity, so that the polynomial in the first equation of (37)

\footnotetext{
${ }^{5}$ The quantity $\left(\beta_{0}+4 / 3 \mu_{0}\right) / \rho_{0}$ is equal to the quantity $\lambda+2 v$ in equations (14) and (23) of [Davis and Brenner 2012], who employ the unconventional designation of $\rho_{0} \lambda$ in their equation (2) as bulk viscosity.
} 
is "well-tempered", that is has derivatives that are all of comparable magnitude for arguments near unity.

Casting (37) in the standard form of a cubic equation in $z=\tilde{k}^{2}$ :

$\mathbb{A} z^{3}+\mathbb{B} z^{2}+\mathbb{C} z+\mathbb{D}=0, \quad$ with

$\mathbb{A}=a c \tilde{s}, \quad \mathbb{B}=(a d+b c-e f) \tilde{s}-e g, \quad \mathbb{C}=(b d-g) \tilde{s}-(e+f) \tilde{s}^{2}, \quad \mathbb{D}=-\tilde{s}^{3}$,

the three roots are given by the well-known formula

$$
\begin{gathered}
z_{k}=-\frac{\mathbb{B}}{3 \mathbb{A}}\left[1+2\left(1-3 \mathbb{A} \mathbb{C} / \mathbb{B}^{2}\right)^{1 / 2} \cos \frac{2 k \pi+\phi}{3}\right] \text { for } k=0,1,2, \\
\text { with } \phi=\cos ^{-1} \zeta, \quad \text { where } \zeta=\frac{1-9 \mathbb{A C} / 2 \mathbb{B}^{2}+27 \mathbb{A}^{2} \mathbb{D} / 2 \mathbb{B}^{3}}{\left(1-3 \mathbb{A} \mathbb{C} / \mathbb{B}^{2}\right)^{-3 / 2}} .
\end{gathered}
$$

The quantities involved in (39) are generally complex, and we can express the complex circular function appearing there in terms of elementary functions as

$$
\begin{aligned}
& \cos \frac{2 k \pi+\phi}{3}=\frac{1}{2}\left(e^{(2 k \pi+\phi) l / 3}+e^{-(2 k \pi+\phi) \imath / 3}\right) \\
& =\frac{1}{2}\left(e^{2 k \pi l / 3}\left[\zeta+\imath \sqrt{1-\zeta^{2}}\right]^{1 / 3}+e^{-2 k \pi l / 3}\left[\zeta-\imath \sqrt{1-\zeta^{2}}\right]^{1 / 3}\right), \quad k=0,1,2,
\end{aligned}
$$

or, by means of yet other well-known formulae [Abramowitz and Stegun 1965, equations 15.1.3-19] in terms of hypergeometric functions $F={ }_{2} F_{1}$ as

$$
\begin{aligned}
& \cos \frac{2 k \pi+\phi}{3}=-\frac{1}{2}\left[\cos \frac{\phi}{3} \mp \sqrt{3} \sin \frac{\phi}{3}\right], \quad \text { for } k=1,2, \quad \text { where } \\
& \cos \frac{\phi}{3}=F\left(-\frac{1}{6}, \frac{1}{6} ; \frac{1}{2} ; 1-\zeta^{2}\right), \quad \sin \frac{\phi}{3}=\frac{1}{3} \sqrt{1-\zeta^{2}} F\left(\frac{1}{3}, \frac{2}{3} ; \frac{3}{2} ; 1-\zeta^{2}\right),
\end{aligned}
$$

with appropriate branch cuts for $\sqrt{1-\zeta^{2}}$ and with $\zeta=\cos \phi$ given by the last equation of (39).

Comparison to the bi-velocity model. We recall that the classical dispersion relation as well as the modification proposed by [Davis and Brenner 2012] involve a quadratic equation for $z$ in lieu of (38). It is clear that such a quadratic arises from (38) for $|\tilde{s}| \ll 1$, which is characteristic of the dissipative regime represented by the previous studies. Indeed, by neglecting terms $O\left(\tilde{s}^{3}\right)$, one obtains a quadratic similar to that given by equation (23) of [Davis and Brenner 2012], with generally different coefficients. This gives a dispersion relation that is quadratic in both $k^{2}$ and $s$ representing a PDE that is quadratic in $\nabla^{2}$ and $\partial_{t}$, for which [Davis and Brenner 2012] offer some special solutions that suggest experiments to arrive at the correct coefficients in the dispersion relations. 


\section{Extension to solids and Cosserat media}

With minor modifications the preceding analysis applies to graded (also known as "higher-gradient") isotropic linear thermo-viscoelastic solids. For this purpose, it suffices to allow for static stress by taking account of (4) and including terms that behave like $s^{-1}$ for $s \rightarrow 0$ in the coefficients $\hat{D}, \ldots, \hat{H}$ in $(7)_{2}$. It can be noted that the coefficient $\hat{D}$ serves to describe both static and dynamic thermoelasticity and, as with the fluids considered above, the static contribution can be included in an equation of state for equilibrium pressure $p_{\mathrm{eq}}(\theta, \rho)$.

Without pursuing the algebraic details, we note that the strain-gradient theory of [Mindlin 1964], anticipated by the seminal works of Piola [dell'Isola et al. 2015], yields dispersion relations for both dilatational and shear waves that are quadratics in $k^{2}$ [Mindlin 1964, (9.34)] with a much simpler form than (32) and (37).

As an extension of [Mindlin 1964], one may treat a more general Cosserat thermo-viscoelasticity by addition to the list of variables in (2) and (5) the Cosserat rotation vector $\vartheta=\left[\vartheta_{i}\right]$ and the moment stress $\boldsymbol{\sigma}^{(2)}=\left[\sigma_{i j}^{(2)}\right]$, conjugate to $\nabla \vartheta$, and by replacing the stress $\sigma$ by a non-symmetric tensor with antisymmetric part defining a vector conjugate to $\vartheta$. Under the rubric of micropolar elasticity, [Eringen 1984] has already given a comprehensive analysis for the isothermal case that leads to a cubic in $k^{2}$ as dispersion relation, and [Abreu et al. 2017] provide a similar analysis with a view to the emerging field of rotational seismology.

Finally, we note that the present type of analysis can be extended to anisotropic media like those considered by [Suiker et al. 2001] by appropriate symmetry restrictions and modification of the relations (6). One possibility is to employ the joint isotropic invariants of the wave vector $\boldsymbol{k}$ and a set of structure tensors to capture the anisotropy [Cowin 1985; Man and Goddard 2016].

\section{Conclusions}

The abstract provides a generally adequate summary of the present work. It is worth emphasizing that the Burnett-order linearized Chapman-Enskog kinetic theory is subsumed by the general wave-number expansions proposed in the present work, which gives more general thermo-viscous response than that of Brenner's bi-velocity model, while also allowing for themo-viscoelastic behavior.

As matter for future work, it would be interesting to consider the utility of nonlocal models in resolving certain fluid-mechanical singularities, such as three-phase contact lines, which bear a certain resemblance to the linear-elastic singularities around crack tips addressed by the non-local elasticity of [Eringen 2002]. 


\section{Appendix: Inverse transforms}

We recall that the inverse Fourier transform of a function $\hat{f}(k)$, with $k^{2}=k_{i} k_{i}$, is a function of $r=|\boldsymbol{x}|$ given by the radial form [Gradshteyn and Ryzhik 2000]

$$
\begin{aligned}
f(\boldsymbol{x}) & =\frac{1}{\sqrt{8 \pi^{3}}} \int e^{\imath \boldsymbol{k} \cdot \boldsymbol{x}} \hat{f}(k) \sin \hat{\vartheta} k^{2} \mathrm{~d} k \mathrm{~d} \hat{\vartheta} \mathrm{d} \hat{\varphi} \\
& =-\frac{1}{\sqrt{2 \pi}} \int_{k=0}^{\infty} \int_{0}^{\pi} e^{\imath k r \cos \hat{\vartheta}} \hat{f}(k) \sin \hat{\vartheta} \mathrm{d} \hat{\vartheta} k^{2} \mathrm{~d} k \\
& =\sqrt{\frac{2}{\pi}} \int_{0}^{\infty} \operatorname{sinc}(k r) \hat{f}(k) k^{2} \mathrm{~d} k, \quad \text { where } \operatorname{sinc}(z)=z^{-1} \sin z
\end{aligned}
$$

One can use (42) to derive the inverse transforms $K(t, \boldsymbol{x})=A(t, \boldsymbol{x}), B(t, \boldsymbol{x}), \ldots$ of the coefficients (8), noting that the functions $f_{1}, f_{2}$ in (19) can be written for $i=1,2$ as

$$
\begin{aligned}
& f_{i}=\left(1+\lambda_{i}^{2} k^{2}\right)^{-1}, \quad \text { where } \quad \lambda_{i}^{2}=b_{i} \lambda_{0}^{2}\left(1+\tau_{1} s\right)^{-1}\left(1+\tau_{2} s\right)^{-1}, \\
& \lambda_{0}^{2}=4 \kappa_{0} \tau_{1} f_{0} / 5, \quad b_{1}=1 / 2, \quad b_{2}=2 / 3, \quad \tau_{1}=\tau_{\sigma 0}, \quad \tau_{2}=\tau_{q 0},
\end{aligned}
$$

Now, the coefficients in (8) can all be expressed as affine forms in $f_{1}, f_{2}$, since

$$
k^{2} f=k^{2}\left(1+\lambda^{2} k^{2}\right)^{-1}=(1-f) / \lambda^{2}, \quad \text { and } \quad f_{1} f_{2}=\frac{b_{1}}{b_{1}-b_{2}} f_{1}+\frac{b_{2}}{b_{2}-b_{1}} f_{2}
$$

First, note that substitution of $\hat{f}=\left(1+\lambda^{2} k^{2}\right)^{-1}$ into (42) gives

$$
f(\boldsymbol{x})=\sqrt{\frac{2}{\pi r^{2}}} \int_{0}^{\infty} \frac{k}{1+\lambda^{2} k^{2}} \sin k r \mathrm{~d} k=\sqrt{\frac{\pi}{2 \lambda^{4} r^{2}}} \exp \left(-\frac{r}{\lambda}\right) .
$$

Second, note that

$$
\begin{aligned}
& \exp \left(-\frac{r}{\lambda}\right)=\exp \left(-\gamma \sqrt{s^{\prime 2}-a^{2}}\right), \\
& \text { where } \quad s^{\prime}=s+\frac{\tau_{1}+\tau_{2}}{2 \tau_{1} \tau_{2}}, \quad a=\frac{\tau_{2}-\tau_{1}}{2 \tau_{1} \tau_{2}}, \quad \gamma=\left(\frac{\tau_{1} \tau_{2}}{b}\right)^{1 / 2} r .
\end{aligned}
$$

However, the inverse Laplace transform [Abramowitz and Stegun 1965]

$$
g(t)=\mathscr{L}^{-1}\left\{\exp \left(-\gamma \sqrt{s^{2}-a^{2}}\right)\right\}=\delta(t-\gamma)+\frac{a \gamma}{\sqrt{t^{2}-\gamma^{2}}} I_{1}\left(a \sqrt{t^{2}-\gamma^{2}}\right) u(t-\gamma),
$$

where $I_{1}(z)$ is the Bessel function of the second kind, $u(t)$ the Heaviside function and $\delta(t)=u^{\prime}(t)$ the Dirac delta, gives

$$
h(t)=\mathscr{L}^{-1}\left\{\exp \left(-\gamma \sqrt{s^{2}-a^{2}}\right)\right\}=\exp \left(-\frac{\tau_{1}+\tau_{2}}{2 \tau_{1} \tau_{2}} t\right) g(t) .
$$


Thus, the coefficients in (20) are seen to involve various powers of $1+\tau_{1} s$ and $1+\tau_{2} s$ multiplying the above transforms, so that the inverse Laplace transform of the resulting products can in principle be obtained by convolution.

\section{Acknowledgement}

I wish to acknowledge several helpful conversations with Professor A. M. J. Davis during the early stages of this work. Also, I am grateful for the hospitality accorded me during extended stays in the Laboratoire PMMH and the Institut Langevin of the École supérieure de physique et chimie industrielles (ESPCI), Paris, where part of this work was completed.

\section{References}

[Abramowitz and Stegun 1965] M. Abramowitz and I. A. Stegun (editors), Handbook of mathematical functions, U.S. National Bureau of Standards, New York, 1965.

[Abreu et al. 2017] R. Abreu, J. Kamm, and A.-S. Reiß, "Micropolar modelling of rotational waves in seismology", Geophys. J. Int 210:2 (2017), 1021-1046.

[Brenner 2009] H. Brenner, "Bi-velocity hydrodynamics: single-component fluids", Internat. J. Engrg. Sci 47:9 (2009), 930-958.

[Brenner 2012] H. Brenner, "Beyond Navier-Stokes", Internat. J. Engrg. Sci 54 (2012), 67-98.

[Cattaneo 1948] C. Cattaneo, "Sulla conduzione de calore", Atti del Semin. Mat. e Fis. Univ. Modena 3:3 (1948), 3-22.

[Chapman and Cowling 1960] S. Chapman and T. G. Cowling, The mathematical theory of nonuniform gases: an account of the kinetic theory of viscosity, thermal conduction, and diffusion in gases, Cambridge University Press, New York, 1960.

[Coleman 1964] B. D. Coleman, "Thermodynamics of materials with memory", Arch. Rational Mech. Anal 17 (1964), 1-46.

[Coleman and Noll 1961] B. D. Coleman and W. Noll, "Foundations of linear viscoelasticity", Rev. Mod. Phys 33 (1961), 239-249.

[Cowin 1985] S. C. Cowin, "The relationship between the elasticity tensor and the fabric tensor", Mech. Materials 4:2 (1985), 137-147.

[Davis and Brenner 2012] A. M. J. Davis and H. Brenner, "Thermal and viscous effects on sound waves: revised classical theory”, J. Acoust. Soc. Am. 132:5 (2012), 2963-2969.

[dell'Isola et al. 2015] F. dell'Isola, U. Andreaus, and L. Placidi, "At the origins and in the vanguard of peridynamics, non-local and higher-gradient continuum mechanics: an underestimated and still topical contribution of Gabrio Piola", Math. Mech. Solids 20:8 (2015), 887-928.

[Eringen 1984] A. C. Eringen, "Plane waves in nonlocal micropolar elasticity", Int. J. of Eng. Sci. 22:8-10 (1984), 1113-1121.

[Eringen 1992] A. C. Eringen, "Vistas of nonlocal continuum physics", Internat. J. Engrg. Sci 30:10 (1992), 1551-1565.

[Eringen 2002] A. C. Eringen, Nonlocal continuum field theories, Springer, 2002.

[Goddard 1992] J. D. Goddard, "History effects in transient diffusion through heterogeneous media", Ind. Eng. Chem. Res. 31:3 (1992), 713-721. 
[Goddard 2010] J. D. Goddard, "On material velocities and non-locality in the thermo-mechanics of continua", Int. J. Eng. Sci. 48:11 (2010), 1279-1288. .

[Goddard and Lee 2017] J. D. Goddard and J. Lee, "On the stability of the $\mu(I)$ rheology for granular flow", J. Fluid Mech 833 (2017), 302-331.

[Gradshteyn and Ryzhik 2000] I. S. Gradshteyn and I. M. Ryzhik (editors), Table of integrals, series, and products, Academic Press, New York, 2000.

[Ignaczak and Ostoja-Starzewski 2009] J. Ignaczak and M. Ostoja-Starzewski, Thermoelasticity with finite wave speeds, Oxford University Press, New York, 2009.

[Man and Goddard 2016] C.-S. Man and J. Goddard, "Remarks on isotropic extension of anisotropic constitutive functions via structural tensors", Math. Mech. Solids 23:4 (2016), 554-563.

[Maxwell 1867] J. C. Maxwell, "On the dynamical theory of gases", Phil. Trans. 157 (1867), 49-88.

[Maxwell 1879] J. C. Maxwell, "Stresses in rarefied gases arising from inequalities of temperature", Phil. Trans. 170 (1879), 231-256.

[Mindlin 1964] R. S. Mindlin, "Micro-structure in linear elasticity", Arch. Ratl. Mech. Anal. 16:1 (1964), 51-78.

[Müller and Ruggeri 1998] I. Müller and T. Ruggeri, Rational extended thermodynamics, 2nd ed., Springer Tracts in Natural Philosophy 37, Springer, 1998.

[Pierce 1981] A. D. Pierce, Acoustics: an introduction to its physical principles and applications, McGraw-Hill, New York, 1981.

[Pierce 1990] A. D. Pierce, "Wave equation for sound in fluids with unsteady inhomogeneous flow", J. Acoust. Soc. Am. 87:6 (1990), 2292-2299.

[Suiker et al. 2001] A. Suiker, A. Metrikine, and R. D. Borst, "Comparison of wave propagation characteristics of the Cosserat continuum model and corresponding discrete lattice models", Int. J. Solids Structs. 38:9 (2001), 1563-1583.

Received 11 Mar 2018. Accepted 24 Jul 2018.

JOE D. GODDARD: jgoddard@ucsd.edu

Department of Mechanical and Aerospace Engineering, University of California, San Diego, 9500 Gilman Drive, La Jolla, CA 92093-0411, United States 
MATHEMATICS AND MECHANICS OF COMPLEX SYSTEMS

EDITORIAL BOARD

ANTONIO CARCATERRA

ERIC A. CARLEN

FRANCESCO DELL'ISOLA

RAFFAELE ESPOSITO

ALBERT FANNJIANG

Gilles A. FrancFort

Pierangelo MARCAT

JEAN-JACQUES MARIGO

Peter A. Markowich

MARTIN OSTOJA-STARZEWSKI

PIERRE SEPPECHER

DAVID J. STEIGMANN

PAUl STEINMANN

Pierre M. SuQuet

MANAGING EDITORS

MICOL AMAR

CORRADO LATTANZIO

ANGela MadeO

MARTIN OSTOJA-STARZEWSKI

ADVISORY BOARD

ADNAN AKAY

HOLM ALTENBACH

MiCOL AMAR

HARM ASKES

TEODOR ATANACKOVIC

VICTOR BERDICHEVSKY

GUY BOUCHITTÉ

ANDREA BRAIDES

ROBERTO CAMASSA

MAURO CARFORE

ERIC DARVE

FELIX DARVE

ANNA DE MAS

Gianpietro Del Piero

EMMANUELE Di BENEDETTO

BERNOLD FIEDLER

IRENE M. GAMBA

DAVID Y. GAO

SERGEy GaVRILYUK

TIMOTHY J. HEALEY

DOMINIQUE JEULIN

ROGER E. KHAYAT

CORRADO LATTANZIO

ROBERT P. LIPTON

ANGELO LUONGO

ANGELA MADEO

JUAN J. MANFREDI

CARLO MARCHIORO

ROBERTO NATALINI

PATRIZIO NEFF

ANDREY PIATNITSKI

ERRICO PRESUTTI

Mario Pulvirent

LuCio Russo

Miguel A. F. Sanjuan

PATRICK SELVADURA

ALEXANDER P. SEYRANIAN

MiRosLaV ŚILHAVY

GUIDO SWEERS

ANTOINETTE TORDESILLAS

LEV TRUSKINOVSKY

JUAN J. L. VELÁZQUEZ

VINCENZO VESPR

ANGELO VULPIANI msp.org/memocs

Università di Roma "La Sapienza", Italia

Rutgers University, USA

(CO-CHAIR) Università di Roma "La Sapienza", Italia

(TREASURER) Università dell'Aquila, Italia

University of California at Davis, USA

(CO-CHAIR) Université Paris-Nord, France

Università dell'Aquila, Italy

École Polytechnique, France

DAMTP Cambridge, UK, and University of Vienna, Austria

(CHAIR MANAGING EDITOR) Univ. of Illinois at Urbana-Champaign, USA

Université du Sud Toulon-Var, France

University of California at Berkeley, USA

Universität Erlangen-Nürnberg, Germany

LMA CNRS Marseille, France

Università di Roma "La Sapienza", Italia

Università dell' Aquila, Italy

Université de Lyon-INSA (Institut National des Sciences Appliquées), France

(CHAIR MANAGING EDITOR) Univ. of Illinois at Urbana-Champaign, USA

Carnegie Mellon University, USA, and Bilkent University, Turkey

Otto-von-Guericke-Universität Magdeburg, Germany

Università di Roma "La Sapienza", Italia

University of Sheffield, UK

University of Novi Sad, Serbia

Wayne State University, USA

Université du Sud Toulon-Var, France

Università di Roma Tor Vergata, Italia

University of North Carolina at Chapel Hill, USA

Università di Pavia, Italia

Stanford University, USA

Institut Polytechnique de Grenoble, France

Università dell'Aquila, Italia

Università di Ferrara and International Research Center MEMOCS, Italia

Vanderbilt University, USA

Freie Universität Berlin, Germany

University of Texas at Austin, USA

Federation University and Australian National University, Australia

Université Aix-Marseille, France

Cornell University, USA

École des Mines, France

University of Western Ontario, Canada

Università dell'Aquila, Italy

Louisiana State University, USA

Università dell'Aquila, Italia

Université de Lyon-INSA (Institut National des Sciences Appliquées), France

University of Pittsburgh, USA

Università di Roma "La Sapienza", Italia

Istituto per le Applicazioni del Calcolo "M. Picone", Italy

Universität Duisburg-Essen, Germany

Narvik University College, Norway, Russia

Università di Roma Tor Vergata, Italy

Università di Roma "La Sapienza", Italia

Università di Roma "Tor Vergata", Italia

Universidad Rey Juan Carlos, Madrid, Spain

McGill University, Canada

Moscow State Lomonosov University, Russia

Academy of Sciences of the Czech Republic

Universität zu Köln, Germany

University of Melbourne, Australia

École Polytechnique, France

Bonn University, Germany

Università di Firenze, Italia

Università di Roma La Sapienza, Italia

MEMOCS (ISSN 2325-3444 electronic, 2326-7186 printed) is a journal of the International Research Center for the Mathematics and Mechanics of Complex Systems at the Università dell' Aquila, Italy.

Cover image: "Tangle” by @ John Horigan; produced using the Context Free program (contextfreeart.org).

\section{PUBLISHED BY}

mathematical sciences publishers

nonprofit scientific publishing

http://msp.org/

(C) 2018 Mathematical Sciences Publishers 
Mathematics and Mechanics of Complex Systems vol. 6 no. 4

A model for interfaces and its mesoscopic limit

Michele Aleandri and Venanzio Di Giulio

Optimal orthotropy and density distribution of two-dimensional structures

Narindra Ranaivomiarana, François-Xavier Irisarri, Dimitri

Bettebghor and Boris Desmorat

A multiphysics stimulus for continuum mechanics bone remodeling 307

Daniel George, Rachele Allena and Yves Rémond

On linear non-local thermo-viscoelastic waves in fluids

Joe D. Goddard

Heterogeneous directions of orthotropy in three-dimensional structures: finite element description based on diffusion equations

Rachele Allena and Christophe Cluzel

A general method for the determination of the local orthotropic directions of heterogeneous materials: application to bone structures using $\mu \mathrm{CT}$ images

Christophe Cluzel and Rachele Allena

MEMOCS is a journal of the International Research Center for the Mathematics and Mechanics of Complex Systems at the Università dell'Aquila, Italy.

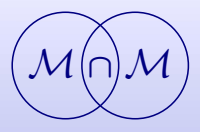

